\title{
A Survey of Optimization Method for Oil-gas Pipeline Network Layout
}

\author{
Fangfang Li, Qiang Liu, Xiu Guo, Jun Xiao \\ School of Information and Control Engineering \\ Liaoning Shihua University \\ Fushun, 113001, China \\ Email: lifang19891npu@163.com
}

\begin{abstract}
Gathering and transferring pipeline network of oil-gas system exerts remarkable impact on construction cost for whole oilfield engineering. Based on analysis of previous optimization methods for oil-gas pipeline network layout, this paper introduces some frequently used optimization design methods and discusses their advantages and disadvantages. Firstly, some classical algorithms are introduced. Subsequently, we discuss the general topology model and the optimization classification of oil-gas pipeline network layout, where the well group division, optimization model and planning algorithms are introduced systematically. Finally, this paper points out the possible development in the areas of oil-gas pipeline network layout.
\end{abstract}

Keyword-oil-gas; pipeline network; layout optimization; intelligent optimization; steiner minimum tree.

\section{INTRODUCTION}

Gathering and transferring pipeline network of oil-gas system plays an important role in oilfield production. Its investments are generally accounted for about 40 percent of the entire oilfield engineering [1]. The optimization design can not only reduce investment but also improve the operation efficiency of the system.

Generally, the pipeline network layout optimization of oil-gas gathering and transferring system includes topology optimization, location optimization and parameter optimization[2]. These optimization problems refer to discrete optimization and parameter optimization problems, moreover, some sub-problems have been proved to be non-deterministic polynomial(NP)-hard [3], which make it much difficult to be solved. In recent years some intelligent optimization algorithms such as genetic algorithm (GA), artificial neural network algorithm (ANN), ant colony algorithm (ACO), particle swarm algorithm (PSO) with its unique advantages are widely used in oil-gas pipeline network optimization, especially in the layout of pipeline network. The optimizations of oil-gas pipeline network layout have been widely studied during the past decades. In 1972, Guoxi Zhang of York University proposed a Steiner algorithm [4]. In 1978, Edgar first used the generalized reduced gradient method to the design of natural gas transferring network[5]. In1990, Simposon, Dandy and Murphy presented GA to the optimization design [6-8]. In 2002, Zhou, Min studied the supply of natural gas distribution network based on the GA [9].In 2004, Chan, Chung, SH applied the standard GA to the distribution network [9]. Despite the research in our country is relatively late, the results are remarkable. In 1998 Shuwen Li studied the optimization of natural gas network and proposed some optimization methods: Kruskal algorithm, Prime algorithm [10]. Yang Liu discussed the optimization of oil-gas pipeline network layout in a given network and issued "Star Topology Optimization Design of N-level Network" [11]. In 2003 he first used GA in field of oil-gas pipeline network [12]. In addition, aimed at the shortcoming of the GA, Jianjun Yang combined GA and simulated annealing algorithm (SAN) to solve the star network topology [13]. In 2002, Jiancheng Leng used ANN to determine multilevel star network [1]. In 2012, Mingyue Sun solved the model based on the integer coding PSO algorithm[14].In2011, Zili Zhang presented a optimization algorithm fitted for the oil-gas gathering network that considering obstacle[15].

In summary, these results have achieved certain economic benefits, but there are a lot of problems have not been completely resolved due to its complexity and diversity. In addition,the optimization techniques that considering obstacle are still in the initial stage. Cost-effective planning algorithms that enable the optimization of oil-gas pipeline network layout still remain an open area of research.

\section{GENERAL TOPOLOGY OF OIL-GA PIPELINE NETWORK}

For the optimization of oil-gas pipeline network layout, the selection of network topology structure determines the success or failure of the whole optimization research directly. At present, the widely used forms of network topology structure are: Multilevel Star (MS), Multistage Star - Ring (MSR), Multistage Star - Tree (MST) [16]. 


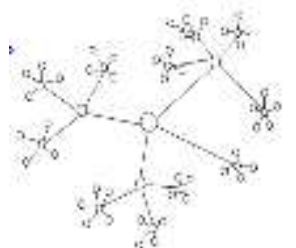

(a)

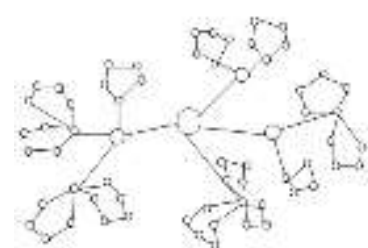

(b)

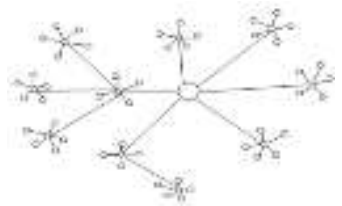

(c)

Figure 1. The topology structure of oil -gas pipeline network (a) MS (b) MSR (c) MST

\section{OIL-GAS PIPELINE NETWORK LAYOUT OPTIMIZATION CLASSIFICATIONS}

\section{A. Well group division}

The design of well group division in the oil-gas pipeline network system is determined by the radius and a certain pit. The methods for the well group division, such as equidistant grid grouping, formatting circular grouping and random circular grouping are actually the division of the collection from the perspective of mathematical. K-Means clustering algorithm [17] is an unsupervised learning algorithm that commonly used in network planning and also can be used to solve classification problems. The operation process of the algorithm is mainly as follow:

a) Choose $\mathrm{K}$ points as the initial centroids.

b) Calculate the distance that each point from the centroid of mass and the point will be added to the nearest cluster.

c) Calculate the average of the coordinates of all points in each cluster and make this value as a new centroid and repeat b), c) until it no longer change .

d) Repeat the above steps until every clustering cluster have reached the ideal state.

The K-Means clustering algorithm used to the well group division is a new attempt. Clustering algorithm is very easy lead to no optimal because it's sensitive to the selection of the initial centroid .

\section{$B$. The review of model and planning algorithms}

The topology structures of oil-gas pipeline network have many kinds, such as MS, MST MSR. In all kinds of topology structure, the Minimum Spanning Tree (MST) connecting more points and has strong expansion and evolution, which has been widely used in the optimization designs.

The MST is an important part of graph theory. The diagram in graph theory is composed of points and edges, similarity, the model of oil-gas pipeline network model can be characterized as diagram. The methods that available through limited search to determine the MST are mainly Prime, Kruskal[10]. If introduction of extra points, the total length of the minimal tree is to be less than or equal to the methods that described above [18].We usually called the extra points Steiner(S) points, obtained the minimum tree is SMT. Thus the topology of oil-gas pipeline network can be transformed into the SMT model.

The SMT problems can be traced back to the early seventeenth Century. Famous mathematician Fermat put out a question: "There are three points on the plane, try to find the fourth point and make the distance to the three points is minimum." In the early 19th century, the famous geometrician Jakbo Steinerto carried out a promotion on this issue: Determine the $n+1$ point so that the distance between the given $\mathrm{n}$ points is the shortest [19]. Here we give a general definition: For the fixed point set in plane $A=\left\{A_{1}, A_{2}, A_{3}, \cdots, A_{n}\right\}$, insert a new point set $S=\left\{S_{1}, S_{2}, S_{3}, \cdots, S_{m}\right\}$, and make a network that connecting these fixed points shortest. The network connecting all fixed points is called the SMT, the insert points are called the $S$ points.

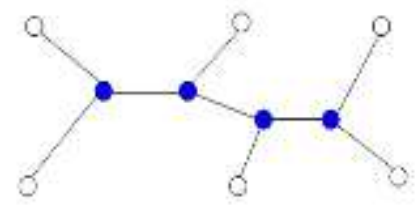

Figure 2. (a) $\bigcirc$ The given points (b) The $\mathrm{S}$ points

SMT is mainly divided into Euclidean Steiner Minimum Tree (ESMT), Rectilinear Steiner Minimum Tree (RSMT) and Graphical Steiner Minimum Tree (GSMT) [20]. According to computational geometry, the SMT is a NP-hard. Considering its NP-hard characteristic, most previous research efforts were put on the approximate algorithm [21]. In recent years, many problems have been solved with the Euclidean Steiner ratio of Gilbert-Polak conjecture [22], the existence of a better approximation algorithm[23-25] and polynomial-time approximation strategies

(PTAS) [26] were put forward, thus making Steiner problems become a very active field.

In the last century of 60 's, Melzak gave an algorithm to solving the ESMT problem [27]. Provan proposed a $\varepsilon$ - approximation algorithm and converted an ESMT problem to GSMT problem [28]. On the other hand, in case of the problems of GSMT, a well-known concept of Complete Graph (CG) and Minimum Spanning Tree (MST) were conjunction used to solve the problems that was presented by Lawler[29]. At present, the best way to solve the ESMT is GeoSteiner algorithm proposed by Warme[30]. So far only a few studies have been applied to the practical, most of which are exponential computations with the increase of point numbers. In the past few years, the intelligent optimization techniques have drawn extensive attention from both domestic and foreign due to the good global search ability and robustness. For the SMT problems, some studies based on these techniques have been developed, such as the GA [31], the PSO algorithms [32] 
and the Evolutionary algorithms [33] for the ESMT problems, the ACO for the GSMT [34], the ACO for the RSMT [35]. Intelligent algorithms have more advantages than the traditional in optimization problems, however, to the best of author's knowledge, so far few research apply the intelligent optimization techniques to solve the Euclidean Steiner Minimal Tree with Obstacles (ESMTO).

The prototype of SMT appeared in every aspect of our lives. Whether in the south to north water diversion project, the west to east gas pipeline project, or the network planning, all of them can be transformed into SMT problems. The difficulty in solving SMT problems is to determine the numbers and locations of the $S$ points, thus searching for effective intelligent algorithm becomes significant.

\section{CONCLUSIONS}

a) The optimization design of oil-gas pipeline network layout is quite difficult due to its complexity and diversity. The optimization of methods, such as dynamic programming method, enumeration method, hierarchical optimization strategy and Steiner algorithm were used for this complex problem.

b) The traditional optimization of oil-gas pipeline network is a computationally difficult and time-consuming process. Thus, the intelligent optimization techniques with good global search ability and robustness have drawn extensive attention and widely used for it.

c) From the published literatures, the optimization methods of oil-gas pipeline network are basically based on the idea of problem decomposition. Although many research efforts have been put on oil-gas pipeline network layout, there are still some problems to be solved, such as the multi-objective optimization considering obstacles and operation cost, the whole optimization considering operation cost and construction cost, and the optimization design taking the loss of operation into account.

\section{ACKNOWLEDGMENT}

This project is supported by PhD Start-up Fund of Liaoning Province of China (Grant No. 20131061) and National Natural Science Foundation of China (Grant No. 51305192).

\section{REFERENCES}

[1].Leng.J. and Liu.Y, The Topology Optimization of Oil \& Gas Gathering and Transportation Network Based on the Neural Network Technique[D], Daqing Petroleum Institute,2002,pp.1-4.

[2].Sun.J. and Liu.Y,The Layout Optimal Design of Annular Oil \& Gas Gathering and Transportation Pipe Networ [D], Daqing Petroleum Institute,2010,pp.2-5.

[3].Yang.H, Optimal Layout of Oil-Gas Gathering and Transportation Pipeline Network Considering Operating Expenses[D],Dalian University of Technology, 2010, pp.1-10.

[4].S.K.Hang, The Generation of Minimal Tree with a Steiner Topology[J],Journal of Association of Computing Machinery,19(4),1972,pp.699-711.
[5].Edgar T.F.Himmelblau D.M, Bickel T.C, Optimal Design of Gas Transmission Networks[J].Society of Petroleum Engineering Journal.SPE Number, 4:6031,1978.

[6].MurphyL.J.,SimposonA.R, Genetic Algorithms in Pipeline Network Optimization[R],Department of Civil and EnvironmenEngineering,1992,pp.9-13.

[7].DandyG.C,Simposon A.R.and Murphy L.J,A review of pipeline network optimization techniques, Proc. Watercomp,1993.

[8].SimposonA.R.,MurphyL.J.,Dandy,G.C, Pipe Optimization Using Genetic Algorithms[J].Proc.Spec.Conf of WaterRes. Planningand Manag,1993,pp.392-395.

[9].Chan,E.T.S and Chung,S.H, Multi criteria Genetic Optimization For Distribution Network Problrms[J]. International Journal of Advanced Manufacturing Technology, 2004, pp.517-532.

[10].Li.S.,Zhang and R.Meng, Pileline layout Optimization of Large Gas Field Gathering [J], Petroleum Planning \&Design ,1998.

[11].Liu.Y, Optimal Design of Getting and Delivering System of Crude Oil,In Optimization and Computing, Elsevier Science Publishers B.V,1990.

[12].Liu.Y and Wei.L, Study on Optimization Technology for Oilfield Surface Pipeline Network Based on Intelligence Computation[D],Daqing Petroleum 2005,pp.28-52.

[13].Yang.J and Zhan.Hand and Liu.Y, The Hybrid Genetic Algorithm For The Star Topology Optimization Of Crude Oil Gathering and Transportation Pipeline Network [J], Journal of Southwest Petroleum University Vol.30,No.4,2008.

[14].Sun.M and Xu.W, Optimal Design Of Tree-type Network Based On Integer Coding Particle Swarm Algorithm[N], Journal of Water Resources \& Water Engineering,Vol.23 No.6 2012.

[15]Zhang.Z, The Optimization and Programming of Oilfield Surface Gathering Network[D],China University of Petroleum(Esat China),2011,pp.1-15.

[16].Wei.L and Liu.Y, Study on Optimization Technology for Oilfield Surface Pipeline Network Based on Intelligence Computation,[D],Daqing Petroleum Institute, 2005, pp. 28-36.

[17].Nguyen and Yao.W, Clustering Algorithm and Hopfield Neural Network Used to Loope Network [J], YellowRiver ,Vol.34,2012.

[18].Bai,J.andLi.Y, Review on Optimal Design of Natural Gas Pipeline Network, Pipeline Technique and Equipment, No.6,2006.

[19].Ho.J.M, Vijayan.G and Wong.C.K, New algorithms for the rectilinear Steiner tree problem.IEEE Transactions on Computer Aided Design, 1990, pp.160 -193.

[20].Kun.Ye, Research on global optimization algorithm for Steiner tree problem based on visualization experiment[D], Henan University of Science and Technology,2012,pp.2-76.

[21]Muller-Hannemann.M and Tazari.S, A near linear time approximation scheme for Steiner tree among obstacles in the plane,Computational Geometry, 2010,pp.395-409.

[22].Du and F. Hwang, A proof of Gilbert-Pollak conjecture on the Steiner ratio.Alg orithmica, No.7,1992,pp.121-135.

[23]A.Zelikovsky, Better Approximation Bounds for the Network and Euclidean Steiner Tree Problems, Technical report CS-96-06,University of Virginia, 1996.

[24].M.Karpinski and A.Zelikovsky, New approximation algorithms for the Steiner tree problem[J],Journal of Combinatorial Optimization, No.1,1997,pp.47-65.

[25].G.Robins and A.Zelikovsky, Tighter bounds for Graph Steiner Tree Approximation, SIAM [J] DiscreteMath, 19(1), 2005,pp.122-134.

[26].S.Arora, Polynomial Time Approximation Schemes for Euclidean and other Geometric Problems, [JACM], 45(5),1998,pp.753-782.

[27].E.Gassner, The Steiner Forest Problem revisited Journal of Discrete Algorithms,2010,pp.154-163.

[28].Provan, J.S, An approximation scheme for finding Steiner trees with obstacles, SIAM Journal of Computing,17 (5), 1998,pp.920-934.

[29].Lawler. E, Combinatorial optimization: networks and matroids, New York: Holt,Rinehart and Winston,1976. 
[30].Winter.P and Zachariasen.M, Euclidean Steiner minimum trees: An improved exact algorithm, Networks, 30(2), 1997,pp.149-166.

[31].Yang.C and Yan.X, A Tree-Coded Hybrid Genetic Algorithm of Minimun Rectilinear Steiner Tree and Its ParallelModel,Microelectronics ,Vol.29,1999.

[32]Liu.Q and Wang.C, Multi-terminal pipe routing by Steiner minimal tree and particle swarm optimisation, Enterprise Information Systems, Taylor \& Francis,Vol.6,2012,pp.316-327.
[33]Yang.B, A hybrid evolutionary algorithm for the Euclidean Steiner tree problem using local searches, Lecture Notes in Computer Science, 2006, pp. 60-67.

[34]Luyet.L, Varone.S.and Zufferey.N, An ant algorithm for the Steiner tree problem in graphs,Lecture Notes in Computer Science, 2007,pp.42-51,

[35]Hu.Y, ACO-Steiner ant colony optimization based rectilinear Steiner minimal tree algorithm[J], Journal of Computer Science \& Technology,2006,pp. 147-152. 